Research Article

\title{
Modelling of Jatropha Oil Hydrocracking in a Trickle-Bed Reactor to Produce Green Fuel
}

\author{
Yuswan Muharam (D), Dianursanti, and Andrey Sapati Wirya \\ Department of Chemical Engineering, Faculty of Engineering, University of Indonesia, Depok 16424, Indonesia \\ Correspondence should be addressed to Yuswan Muharam; muharam@che.ui.ac.id
}

Received 15 April 2021; Revised 1 September 2021; Accepted 2 November 2021; Published 9 December 2021

Academic Editor: Alireza Baghban

Copyright (c) 2021 Yuswan Muharam et al. This is an open access article distributed under the Creative Commons Attribution License, which permits unrestricted use, distribution, and reproduction in any medium, provided the original work is properly cited.

\begin{abstract}
Trickle-bed reactor (TBR) modelling to produce green fuel via hydrocracking of jatropha oil using silica-alumina-supported Ni-W catalysts was performed in this research. The objectives of this study are to obtain a TBR with good heat transfer and the optimum condition for high purities of products. A two-dimensional axisymmetric model with a diameter of $0.1 \mathrm{~m}$ and a length of $10 \mathrm{~m}$ was used as a representative of the actual TBR system. Heterogeneous phenomenological models were developed considering mass, energy, and momentum transfers. The optimisation was conducted to obtain the highest green fuel purity by varying catalyst particle diameter, inlet gas velocity, feed molar ratio, and inlet temperature. The simulation shows that a TBR with an aspect ratio of 100 has achieved a good heat transfer. The diesel purity reaches $44.22 \%$ at $420^{\circ} \mathrm{C}$, kerosene purity reaches $21.39 \%$ at $500^{\circ} \mathrm{C}$, and naphtha purity reaches $25.30 \%$ at $500^{\circ} \mathrm{C}$. The optimum condition is reached at the catalyst diameter of $1 \mathrm{~mm}$, the inlet gas velocity of $1 \mathrm{~cm} / \mathrm{s}$, the feed molar ratio of 105.5 , and the inlet temperature at $500^{\circ} \mathrm{C}$ with the green fuel purity of $69.4 \%$.
\end{abstract}

\section{Introduction}

In recent years, the development of renewable fuel sources has become a focus in the science and energy sectors. Fossil fuels (such as gasoline and diesel fuel) have been the primary fuel sources in the industry and transportation sectors. However, fossil fuels are limited energy sources that can run out at any time, which could consequently induce a global energy crisis. Notably, the emission of combustion gases in the form of greenhouse gases and the price of fossil fuels are increasingly expensive. Therefore, it is necessary to develop renewable energy sources to tackle the energy crisis.

Interest in biodiesel and green fuel production has constantly increased over the past decades [1]. For example, Neste Oil in Europe and UOP Honeywell in America have built a hydrogenation-based plant to convert vegetable oil into biodiesel and green fuel [2]. The resulting green fuel should have properties similar to petroleum diesel that can be used in conventional machines.

Green fuel, which is a second-generation renewable fuel, can be produced from vegetable oils, one of which is jatropha oil. Jatropha oil is derived from Jatropha curcas Linn. The plant is spread across several regions in Indonesia with different names such as kalekhe paghar, paku kase, and bindalo. Until now, in Indonesia, jatropha has been used as a traditional medicine and is planted as a living fence. Indonesian climate favours the growth of this plant; therefore, the use of jatropha as a source of renewable energy in Indonesia is possible [3].

Large-capacity green fuel production is performed using hydrotreating and hydrocracking lines, which involve three phases. The raw material is the liquid phase, the catalyst is the solid phase, and hydrogen is the gas phase. There are three types of reactors for three-phase processes: stirred slurry reactors, slurry bubble column reactors, and tricklebed reactors (TBRs). In slurry reactors, the liquid and solid phases are mixed so that, at the end of the reaction, the separation of the products and catalyst is required as well as the additional cost for separation. Furthermore, abrasion of the catalyst may also occur in slurry reactors [4]. Slurry reactors are generally used for fermentation because the solid and liquid phases are mixed. The TBR is a three-phase reactor that uses a stream in trickle regime. In TBR, gas flows in the same or opposite direction with liquid over a packed 
bed consisting of catalyst particles [5]. Therefore, TBRs are chosen based on their more efficient operation than slurry reactors. However, the problem often encountered in using TBRs is poor heat transfer. As a result, the optimisation of heat control is required [6].

The TBR model has been successfully used in various processes to predict the process variables and is still being developed till date. Giri and Majumder [7] reported the influences of concurrent downflow of air-Newtonian and non-Newtonian liquid systems on pressure drop and on its reduction in a downflow TBR. Observably, the reactor's efficiency may be influenced by the nonideality factor, which is higher than the pulse and trickle-flow regimes. Al-Naimi et al. [8] studied the hydrodynamics of TBRs in nonambient conditions for air-water and air-acetone systems, which represent pure organic liquids with low surface tension. Their study showed that the pressure drop increases with increasing superficial gas and liquid velocity, while it decreases with increasing bed temperature. Russo et al. [9] developed a dynamic model of a nonisothermal TBR for sugar hydrogenation. The resulting model can be applied to various reaction systems and tested to obtain both qualitatively and quantitatively promising results. Muharam et al. [10] modelled a TBR for the partial hydrogenation of polyunsaturated fatty acid methyl esters using a steady-state heterogeneous two-dimensional axisymmetric model comprising mass balances in each phase, energy balance, and two-phase Darcy equation-based momentum balance. Methyl linoleate was considered a polyunsaturated fatty acid methyl ester representative, and cis-methyl oleate, transmethyl oleate, and methyl stearate were considered hydrogenated fatty acid methyl esters.

Studies on green fuel production through hydrocracking, hydrotreating, and other pathways, such as biological treatment with the Fischer-Tropsch process, have been conducted. Milbrandt et al. [11] reported that green fuel could be produced from high-temperature biomass gasification, which converted biomass to syngas and then catalytically converted it to liquid and wax products through the Fischer-Tropsch process. Attanatho [12] conducted a study on the hydrotreatment of bio-oils using a microreactor, which could achieve the complete conversion of fatty acid hydrotreating reaction at a temperature of $325^{\circ} \mathrm{C}$. Muharam and Soedarsono [13] observed the hydrodeoxygenation of vegetable oils to produce renewable diesel in a TBR. It has been found that the dominant reacting compounds at temperatures above $310^{\circ} \mathrm{C}$ and a pressure of 15 bar are $\mathrm{C}_{18}$ hydrocarbons. Anand et al. [14] conducted a study on kinetics, thermodynamics, and the reaction mechanism of green fuel production via a hydrocracking system in a fixedbed reactor. However, few studies have developed mathematical models by utilising experimental results to simulate the process on a large scale $[12,15,16]$.

This study simulates the process of jatropha oil hydrocracking in a TBR comprising several tubes in which the solid particles of the catalyst in pellet form are arranged as a so-called fixed bed. The tubes are located in a shell in which the cooling media circulate. The reactions in the tube catalyst beds are exothermic, with the reaction rate heavily influenced by the local temperature. A good design reduces the possibility of hotspots being formed within the reactor. Therefore, the purpose of this study is to obtain a TBR model for the hydrocracking process, a reactor size with good heat transfer, and optimum conditions for high purity green fuel.

\section{Modelling}

To study the jatropha oil hydrocracking process in a TBR, a model was developed to represent the actual condition of a reactor system. A simulation was performed to obtain the solution of the developed model. The use of a model and a simulation can avoid risks and reduce operational costs. Furthermore, this method allows for a detailed study of the most important variables to system performance.

The system modelled in this study is illustrated in Figure 1. A triglyceride solution and gaseous hydrogen enter from the top of the reactor and flow downwards over the packed bed of catalyst particles. In this model, a tube is selected as the representative of all the tubes within a shell based on the assumption that the other tubes undergo the same phenomena as the representative. This is achieved when the temperature of the cooling medium inside the shell is uniform.

The model comprises two parts: the reactor scale and the catalyst particle scale. The reactor scale model was developed based on 2D axisymmetric geometry, with a diameter of $0.1 \mathrm{~m}$ and a length of $10 \mathrm{~m}$, using the concept of computational fluid dynamic (CFD). This CFD method allows for the evaluation of velocity, pressure, temperature, and the species concentration of fluid flow throughout the reactor domain. In addition, this model considers momentum, mass, and energy balances. The catalyst scale model considers radial mass transfer. The kinetic model used is adopted from the kinetic model for the hydrocracking reactions developed by Anand et al. [14], where the reactants are hydrogen and triglyceride. The main products are green fuels comprising diesel (C15-C18), kerosene (C9-C14), and naphtha $(<\mathrm{C} 9)$. Jatropha oil is represented by triglycerides, while the catalyst used is $\mathrm{Ni}-\mathrm{W} / \mathrm{Si}_{2} \mathrm{O}_{3}-\mathrm{Al}_{2} \mathrm{O}_{3}$.

2.1. Momentum Transfer. The momentum transfer is modelled using Darcy's law approach for two phases, which is a continuity equation combined with Darcy's law:

$$
\frac{1}{r} \frac{\partial\left(r c_{1} U_{r}\right)}{\partial r}+\frac{\partial\left(c_{1} U_{z}\right)}{\partial z}=\frac{1}{r} \frac{\partial}{\partial r}\left(r D_{c} \frac{\partial c_{1}}{\partial r}\right)+\frac{\partial}{\partial z}\left(D_{c} \frac{\partial c_{1}}{\partial z}\right)
$$

where $D_{c}$ is the capillary diffusion coefficient. Axial superficial velocity $U_{z}$ and radial superficial velocity $U_{r}$ are described by

$$
\begin{aligned}
& U_{z}=-\frac{\kappa}{\mu} \frac{\partial p}{\partial z} \\
& U_{z}=-\frac{\kappa}{\mu} \frac{\partial p}{\partial r}
\end{aligned}
$$




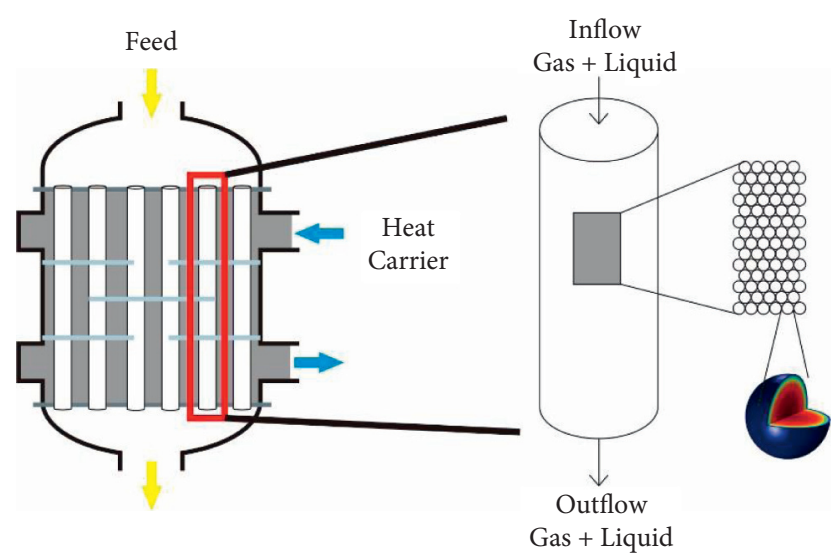

Figure 1: Trickle-bed reactor modelling strategy.

where $\kappa$ is the bed permeability, $p$ is the pressure, and $\mu$ is the average viscosity.

Liquid phase content $c_{1}$ is explained as $c_{1}=\rho_{1} s_{1}$, where $\rho_{1}$ is the liquid phase density and $s_{1}$ is the liquid phase saturation.

The relationship between the saturation of Phases 1 and 2 and the vacant volume in the bed are expressed in

$$
\begin{array}{r}
s_{1}=\frac{\varepsilon_{1}}{\varepsilon_{p}}, \\
s_{2}=\frac{\varepsilon_{2}}{\varepsilon_{p}}, \\
s_{1}+s_{2}=1,
\end{array}
$$

where $\varepsilon_{p}$ is the bed porosity and $\varepsilon_{1}$ and $\varepsilon_{2}$ are the liquid and gas holdups. by

The average physical properties of the mixture are given

$$
\begin{aligned}
& \rho=s_{1} \rho_{1}+s_{2} \rho_{2}, \\
& \frac{1}{\mu}=s_{1} \frac{\kappa_{r 1}}{\mu_{1}}+s_{2} \frac{\kappa_{r 2}}{\mu_{2}} .
\end{aligned}
$$

The superficial fluid velocity is the sum of the superficial velocities of each phase, $U_{1}$ and $U_{2}$, which are correlated using the saturation of the two fluids, as in

$$
\begin{aligned}
U & =U_{1}+U_{2}, \\
U_{1} & =s_{1} U, \\
U_{2} & =s_{2} U .
\end{aligned}
$$

The relative permeabilities of (8) are based on the research results of Sáez and Carbonell [17]:

$$
\begin{aligned}
& \kappa_{r 1}=\delta_{1}^{2.43}, \\
& \kappa_{r 2}=s_{2}^{4.80} .
\end{aligned}
$$

Parameter $\delta_{1}$ is the reduced saturation of Phase 1 (liquid), which is mathematically defined by

$$
\delta_{1}=\frac{\varepsilon_{p} s_{1}-\varepsilon_{1}^{0}}{\varepsilon_{p}-\varepsilon_{1}^{0}} .
$$

In (14), $\varepsilon_{1}^{0}$ is the static holdup of Phase 1 . The equation for calculating $\varepsilon_{1}^{0}$ follows Sáez and Carbonell [17]:

$$
\varepsilon_{1}^{0}=\frac{1}{\left(20+0.9 E \ddot{o}^{*}\right)},
$$

where $E \ddot{o}^{*}$ is the modified Eotvos number, which is expressed as follows:

$$
E \ddot{o}^{*}=\frac{\rho_{1} g d_{p}^{2} \varepsilon_{p}^{2}}{\sigma_{1}\left(1-\varepsilon_{p}\right)^{2}},
$$

where $d_{p}$ is the catalyst diameter and $g$ is the gravity constant.

Bed permeability $(\kappa)$ can be calculated using a slightly modified Ergun equation, as suggested by Propp et al. [18]:

$$
\kappa=\left(\frac{180\left(1-\varepsilon_{p}\right)^{2}}{d_{p}^{2} \varepsilon_{p}^{3}}+\frac{1.8\left(1-\varepsilon_{p}\right) \rho U_{0}}{\mu d_{p} \varepsilon_{p}^{3}}\right)^{-1},
$$

where $U_{0}$ is the inlet mixed superficial velocity.

The capillary diffusion coefficient $\left(D_{c}\right)$ in (1) is a function of capillary pressure:

$$
D_{c}=\frac{\kappa_{r 1} \kappa}{\mu_{1}}\left(s_{1}-1\right) \frac{\partial p_{c}}{\partial s_{1}},
$$

where $p_{c}$ is the capillary pressure, that is, the difference between the gas phase and liquid phase pressure. Capillary pressure is calculated using the equation proposed by Leverett [19]:

$$
p_{c}=\frac{\varepsilon_{p}}{\kappa} \sigma J\left(s_{1}\right)
$$

where $\sigma$ is the gas-liquid surface tension and $J\left(s_{1}\right)$ is a function of capillary pressure on the liquid phase saturation $\left(s_{1}\right)$. The function $J$ is as suggested by Grosser et al. [20]:

$$
J=0.48+0.036 \ln \left(\frac{s_{2}}{s_{1}}\right) \text {. }
$$

2.2. Mass Transfer. The mass transfer in the gas phase uses the following dispersion-convection equation:

$$
\begin{aligned}
\left(\frac{1}{r} \frac{\partial}{\partial r}\left(r D_{G r} \frac{\partial C_{G, i}}{\partial r}\right)+\frac{\partial}{\partial z}\left(D_{G r} \frac{\partial C_{G, i}}{\partial z}\right)\right) & -\left(u_{G r} \frac{\partial C_{G, i}}{\partial r}+u_{G z} \frac{\partial C_{G, i}}{\partial z}\right) \\
& =R_{G L, i},
\end{aligned}
$$

where $C_{G, i}$ is the species $i$ concentration in the gas phase, $D_{G r}$ and $D_{G z}$ are the gas phase dispersion coefficients in the radial and axial directions, $u_{G r}$ and $u_{G z}$ are the gas phase 
interstitial velocities in the radial and axial directions, and $R_{G L, i}$ is the gas-liquid mass transfer rate of species $i$.

The gas-phase interstitial velocities are correlated with the Darcy total velocities in

$$
\begin{gathered}
u_{G}=\frac{U s_{2}}{\varepsilon_{p}}, \\
u_{G r}=\frac{U_{r} s_{2}}{\varepsilon_{p}}, \\
u_{G z}=\frac{U_{z} s_{2}}{\varepsilon_{p}} .
\end{gathered}
$$

The gas-liquid mass transfer rate is expressed in

$$
R_{G L, i}=k_{G L, i} a\left(\frac{p_{G, i}}{H_{c, i}}-C_{i}\right)
$$

where $k_{G L, i}$ is the gas-liquid mass transfer coefficient, $a$ is the gas-liquid interphase specific surface area, $p_{G, i}$ is the partial pressure of species $i$ in the gas phase, and $H_{c, i}$ is Henry's constant of species $i$. $C_{i}$ is the concentration of species $i$ in the liquid phase.

The liquid phase mass transfer uses the following convection-dispersion equation:

$$
\begin{aligned}
& \left(\frac{1}{r} \frac{\partial}{\partial r}\left(r D_{L r} \frac{\partial C_{L, i}}{\partial r}\right)+\frac{\partial}{\partial z}\left(D_{L z} \frac{\partial C_{L, i}}{\partial z}\right)\right)-\left(u_{L r} \frac{\partial C_{L, i}}{\partial r}+u_{L z} \frac{\partial C_{L, i}}{\partial z}\right) \\
& =R_{L S, i}-R_{G L, i}
\end{aligned}
$$

where $C_{L, i}$ is the species $i$ concentration in the liquid phase, $D_{L r}$ and $D_{L z}$ are the liquid-phase dispersion coefficients in the radial and axial directions, $u_{L r}$ and $u_{L z}$ are the liquidphase interstitial velocities in the radial and axial directions, and $R_{L S, i}$ is the liquid-solid mass transfer of species $i$.

The liquid-solid mass transfer rate is expressed in

$$
R_{L S, i}=k_{L S, i} a_{S}\left(C_{i}-C_{p e, i}\right)
$$

where $k_{L S, i}$ is the liquid-solid mass transfer coefficient and $a_{S}$ is the catalyst specific surface area.

Since spherical catalyst particles are used, their specific surface area is as in

$$
a_{S}=\frac{6}{d_{p}}\left(1-\varepsilon_{p}\right)
$$

The liquid-phase interstitial velocities are calculated using

$$
\begin{gathered}
u_{L}=\frac{U s_{1}}{\varepsilon_{p}}, \\
u_{L r}=\frac{U_{r} s_{1}}{\varepsilon_{p}}, \\
u_{L z}=\frac{U_{z} s_{1}}{\varepsilon_{p}} .
\end{gathered}
$$

The mass transfer in the catalyst particles uses the following reaction-diffusion equation:

$$
\frac{1}{r^{2}} \frac{\partial}{\partial r}\left(-r^{2} D_{p e, i} \frac{\partial C_{p e, i}}{\partial r}\right)=R_{p e, i}
$$

where $C_{p e, i}$ is the species $i$ concentration in the catalyst particles, $D_{p e, i}$ is the effective diffusion coefficient of species $i$ in the catalyst particles, and $R_{p e, i}$ is the surface reaction rate of species $i$ in the catalyst particles.

The effective diffusion coefficient is calculated based on the Millington and Quirk model, as shown in

$$
D_{p e, i}=\varepsilon_{p e}^{4 / 3} D_{L, i} \text {. }
$$

The correlations for calculating the mass transfer coefficients are listed in Table 1.

2.3. Energy Transfer. The energy transfer is calculated on the reactor scale by assuming that the thermal equilibrium in all phases is present so that the temperature difference between the phases is negligible. For the energy balance, the equation is given as follows:

$$
\rho C_{p}\left(u_{r} \frac{\partial T}{\partial r}+u_{z} \frac{\partial T}{\partial z}\right)-\left(\frac{1}{r} \frac{\partial}{\partial r}\left(r k_{e r} \frac{\partial T}{\partial r}\right)+\frac{\partial}{\partial z}\left(k_{e z} \frac{\partial T}{\partial z}\right)\right)=Q_{b e d}
$$

where $T$ is the temperature, $\rho$ is the mixed fluid density, $C_{p}$ is the effective heat capacity, $k_{e r}$ and $k_{e z}$ are the thermal conductivities of the mixed fluid in the radial and axial directions, $u_{r}$ and $u_{z}$ are the mixed interstitial velocities in the radial and axial directions, and $Q_{b e d}$ is the reaction heat.

The axial heat capacity and the axial effective thermal conductivity are calculated based on the average volume of each phase using

$$
\begin{aligned}
\left(\rho C_{p}\right)_{e f f} & =\left(1-\varepsilon_{p}\right) \rho_{S} C_{p S}+\varepsilon_{G} \rho_{G} C_{p G}+\varepsilon_{L} \rho_{L} C_{p L}, \\
k_{e z} & =\left(1-\varepsilon_{p}\right) k_{p}+\varepsilon_{L} k_{L}+\varepsilon_{G} k_{G},
\end{aligned}
$$

where $C_{p S}, C_{p G}$, and $C_{p L}$ are the solid, gas, and liquid heat capacities, $\rho_{S}, \rho_{G}$, and $\rho_{L}$ are the solid, gas, and liquid densities, and $k_{p}, k_{L}$, and $k_{G}$ are the solid, liquid, and gas thermal conductivities.

The mixed interstitial velocities for the heat transfer are calculated using

$$
\begin{aligned}
& u_{r}=\frac{U_{r}}{\varepsilon_{p}}, \\
& u_{z}=\frac{U_{z}}{\varepsilon_{p}} .
\end{aligned}
$$

The correlations for calculating the heat transfer coefficients, the thermal conductivity, and the formulas for physical properties are listed in Table 1.

2.4. Kinetic Models. The kinetics used in this study are from the kinetic model developed by Anand et al. [14] based on 


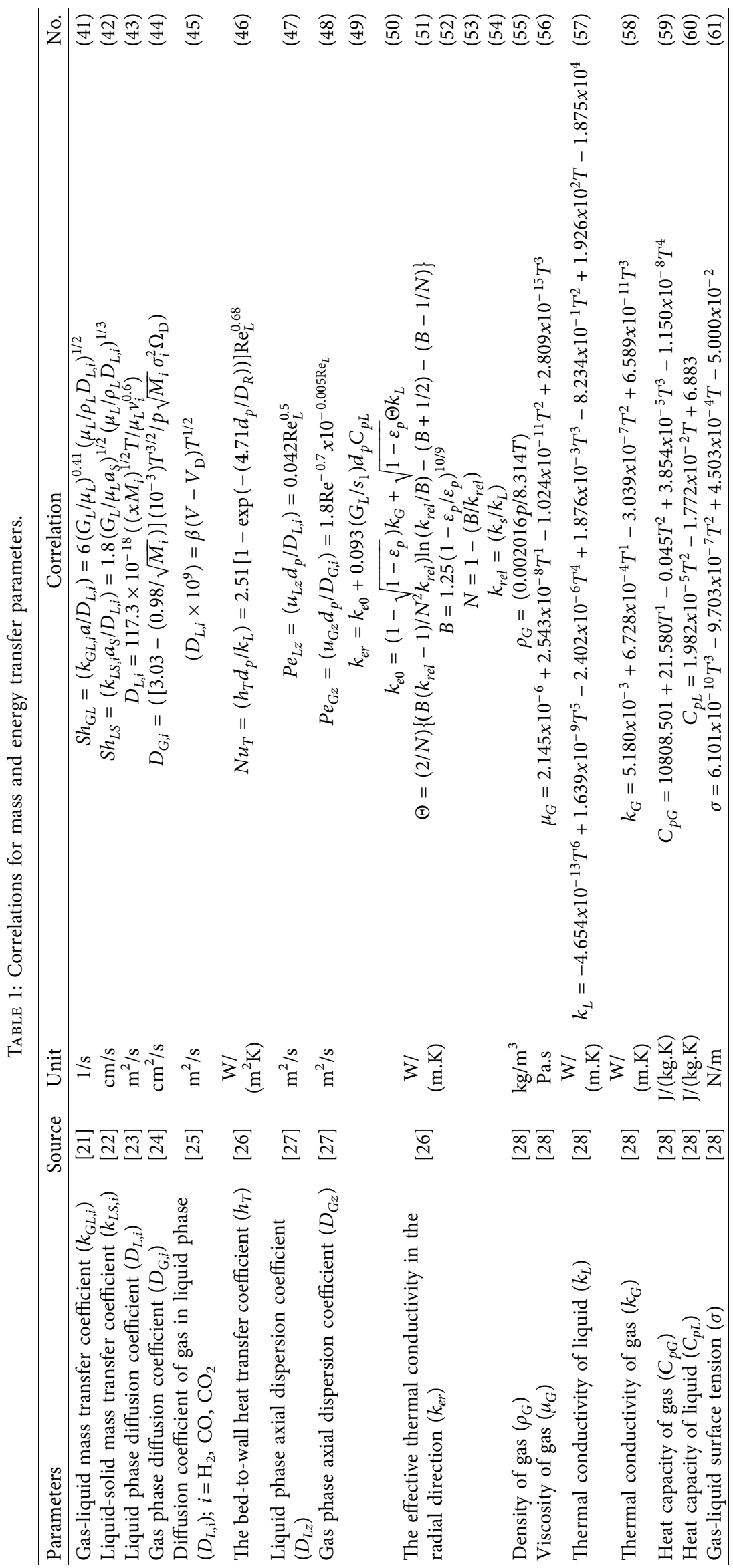


the reactions and the reaction rate coefficients' formulas, as listed in Table 2. The preexponential factors and activation energies for each reaction rate coefficient are shown in Table 3. The vegetable oil is represented by a model compound, i.e., triglycerides. The reactions involve ten components: triglyceride (TG), diesel $\left(\mathrm{C}_{17} \mathrm{H}_{36}\right)$, undecane $\left(\mathrm{C}_{11} \mathrm{H}_{24}\right)$, nonane $\left(\mathrm{C}_{9} \mathrm{H}_{20}\right)$, octane $\left(\mathrm{C}_{8} \mathrm{H}_{18}\right)$, hexane $\left(\mathrm{C}_{6} \mathrm{H}_{14}\right)$, propane $\left(\mathrm{C}_{3} \mathrm{H}_{8}\right)$, wax $\left(\mathrm{C}_{19} \mathrm{H}_{40}\right), \mathrm{CO}_{2}$, and $\mathrm{H}_{2}$. The reactions took place on $\mathrm{Ni}-\mathrm{W} / \mathrm{Si}_{2} \mathrm{O}_{3}-\mathrm{Al}_{2} \mathrm{O}_{3}$ catalyst. In the reaction mechanism, triglycerides react with hydrogen to produce hexane, nonane, diesel, propane, and wax. Some resulting diesel undergoes cracking into kerosene (nonane and undecane) and naphtha (hexane and octane). Wax is a byproduct of the hydrocracking reaction.

2.5. Boundary Conditions. The flow rate at the reactor inlet and the pressure at the reactor outlet were specified for the momentum transfer. For mass transfer, the boundary condition has a mass flux at the reactor inlet and an outflow at the reactor outlet. For heat transfer, the boundary condition has an inlet temperature at the reactor inlet and an outflow at the reactor outlet. At the reactor wall, all boundary conditions have no flux, except that of the energy transfer, where energy flux due to heat transfer from the catalyst bed to the reactor wall was considered.

The boundary conditions for the catalyst pellet model are as follows:

$$
\begin{aligned}
& \text { At } r=0, \\
& \qquad \frac{\partial C_{p e, i}}{\partial r}=0 . \\
& \text { At } r=R_{p}, \quad-D_{p e, i} \frac{\partial C_{p e, i}}{\partial r}=R_{L S, i} .
\end{aligned}
$$

\section{Simulation and Discussion}

One of the problems with TBRs is the high heat transfer resistance in the radial direction of the reactor bed. Therefore, a high ratio of the length to the diameter of the reactor bed or aspect ratio helps reduce the problem. In this study, an aspect ratio of 100 was used to observe the temperature distribution in the reactor bed. The hydrocracking process was simulated in excess hydrogen. To prevent catalyst deactivation, a $5 \% \mathrm{w} / \mathrm{w}$ triglyceride (TG) in diesel was fed to the reactor. The feed is eventually a mixture of gaseous hydrogen and TG solution and enters the reactor at $420^{\circ} \mathrm{C}$. Since the reaction is exothermic, the reactor bed is cooled by a cooling medium, providing the reactor wall with a temperature of $420^{\circ} \mathrm{C}$. The process parameters used in this simulation are listed in Table 4 . The simulation was performed numerically using COMSOL Multiphysics 5.3.

3.1. Temperature Profile. Figure 2 shows the temperature profile in the bed reactor. The increasing temperature in the reactor bed towards the bed outlet indicates that the hydrocracking reaction is exothermic. The heat generated along the reactor is shown in Figure 3. The highest reaction heat occurs in the reactor inlet due to the high concentrations of the reactants in this location. Towards the reactor outlet, the triglyceride concentration decreases as the reactant is converted. The heat generated in the reactor is transported by the flowing fluid to the reactor outlet so that although the reaction heat in the downstream reactor is lower, its temperature is higher. The temperature gradient in the radial direction is shown in Figure 4. The temperature difference from the symmetry axis to the reactor wall increases towards the reactor outlet. Near the reactor inlet, there is a negative gradient. This is because the wall temperature near the reactor inlet is higher than that at the reactor symmetry axis. The temperature gradient is due to resistance to conduction and convection heat transfers in the radial direction. Heat carried downstream of the reactor by the fluid flow accumulates at the reactor outlet so that the temperature at the symmetry axis is higher than that at the reactor wall. The temperature difference between the wall and the symmetry axis at the reactor outlet is $0.47 \mathrm{~K} / \mathrm{m}$ or $0.0235 \mathrm{~K}$, along with a radial distance of $5 \mathrm{~cm}$. It can be seen that the temperature is almost unchanged radially. This indicates that an aspect ratio of 100 provides effective radial heat transfer.

3.2. Concentration Profiles. Figure 5 shows the concentration profile of triglyceride, diesel, and hydrogen. Figure 5(a) shows that triglyceride concentration decreases towards the reactor because it reacts in the reactor to become products. Conversely, the diesel concentration increases towards the reactor outlet (Figure 5(b)). The triglyceride and diesel concentrations are evenly distributed in the radial direction. This proves that the mixing and cooling processes are very effective. The hydrogen concentration increases towards the outlet (Figure 5(c)). This is due to the mass transfer of hydrogen from the gas phase into the liquid phase. This hydrogen situation causes hydrogen molecules to accumulate towards the outlet. Therefore, its concentration is the highest at the reactor outlet. However, the hydrogen transfer to the liquid phase insignificantly reduces its concentration in the gas phase. The hydrogen concentration profile shows that the hydrogen concentration in the liquid phase is constant from the $3 \mathrm{~m}$ position at the reactor inlet to the reactor outlet. This indicates that the hydrogen concentration in the liquid phase is saturated from the $3 \mathrm{~m}$ position.

The concentration profiles in the catalyst particles are shown in Figure 6. These profiles were captured from the particles in the middle of the reactor length and in the symmetry axis of the reactor. Overall, the product concentrations decrease from the core of the catalyst particle towards the external surface of the catalyst particle. The diesel concentration increases until it peaks at the $0.8 \mathrm{~m}$ dimensionless positions of the particles and then decreases towards the external surface of the catalyst particle.

3.3. Optimisation. In this study, optimisation was conducted with the purity of green fuel products as an objective function (i.e., the highest purity). Decision variables are 
TABLE 2: Hydrocracking reactions and their reaction rate coefficients' formula.

\begin{tabular}{lccc}
\hline Reaction rate & Reaction stoichiometry & Reaction rate coefficient & Reaction rate formula \\
\hline$r_{1}$ & $\mathrm{TG}+\mathrm{H}_{2} \longrightarrow$ hexane $+\mathrm{CO}_{2}$ & $k_{1}$ & $k_{1} C_{T G}$ \\
$r_{2}$ & $\mathrm{TG}+\mathrm{H}_{2} \longrightarrow$ nonane $+\mathrm{CO}_{2}$ & $k_{2}$ & $k_{2} C_{T G}$ \\
$r_{3}$ & $\mathrm{TG}+\mathrm{H}_{2} \longrightarrow$ diesel + propane $+\mathrm{CO}_{2}$ & $k_{3}$ & $k_{3} C_{T G}$ \\
$r_{4}$ & $\mathrm{TG}+\mathrm{H}_{2} \longrightarrow$ wax $+\mathrm{CO}_{2}$ & $k_{4}$ & $k_{5} C_{T G}$ \\
$r_{5}$ & Diesel $+\mathrm{H}_{2} \longrightarrow$ nonane + octane & $k_{5}$ & $k_{6} C_{C 9}$ \\
$r_{6}$ & Nonane $+\mathrm{H}_{2} \longrightarrow$ hexane + propane & $k_{6}$ & $k_{7} C_{C 17}$ \\
$r_{7}$ & Diesel $+\mathrm{H}_{2} \longrightarrow$ hexane + undecane & $k_{7}$ & \\
\hline
\end{tabular}

Table 3: Preexponential factors and activation energies for each reaction rate coefficient.

\begin{tabular}{lcr}
\hline Reaction rate coefficient & Preexponential factor $(1 / \mathrm{h})$ & Activation energy $(\mathrm{kJ} / \mathrm{mol})$ \\
\hline$k_{1}$ & $4.65 \times 10^{9}$ & 130 \\
$k_{2}$ & $9.48 \times 10^{8}$ & 122 \\
$k_{3}$ & $1.00 \times 10^{10}$ & 116 \\
$k_{4}$ & $1.02 \times 10^{10}$ & 115 \\
$k_{5}$ & $9.48 \times 10^{8}$ & 122 \\
$k_{6}$ & $4.65 \times 10^{9}$ & 130 \\
$k_{7}$ & $4.65 \times 10^{9}$ & 130 \\
\hline
\end{tabular}

TABle 4: Process parameters.

\begin{tabular}{lc}
\hline Reactor outlet pressure & $80 \mathrm{bars}$ \\
Inlet temperature & $420^{\circ} \mathrm{C}$ \\
Feed molar ratio $\mathrm{H}_{2} / \mathrm{TG}$ & 105.5 \\
Inlet gas velocity & $5 \mathrm{~cm} / \mathrm{s}$ \\
TG fraction in the feed & $5 \% \mathrm{w} / \mathrm{w}$ \\
Wall temperature & $420^{\circ} \mathrm{C}$ \\
\hline
\end{tabular}

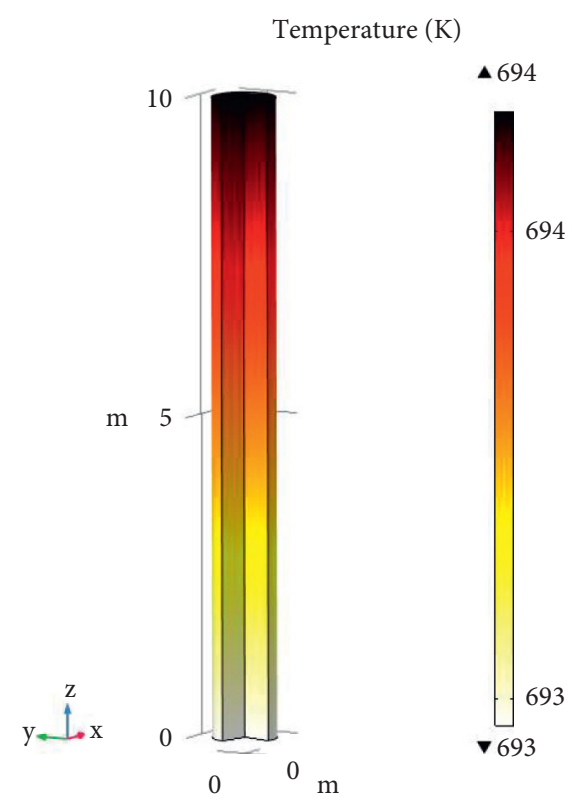

FIgURE 2: Temperature profile.

catalyst pellet diameter, inlet gas velocity, feed molar ratio, and inlet temperature. Based on the base case simulation, the reactor geometry provided good heat transfer so that to accelerate the convergence of the numerical calculations, the optimisation was conducted in isothermal conditions. The range of each parameter is listed in Table 5. The optimisation results are shown in Figure 7.

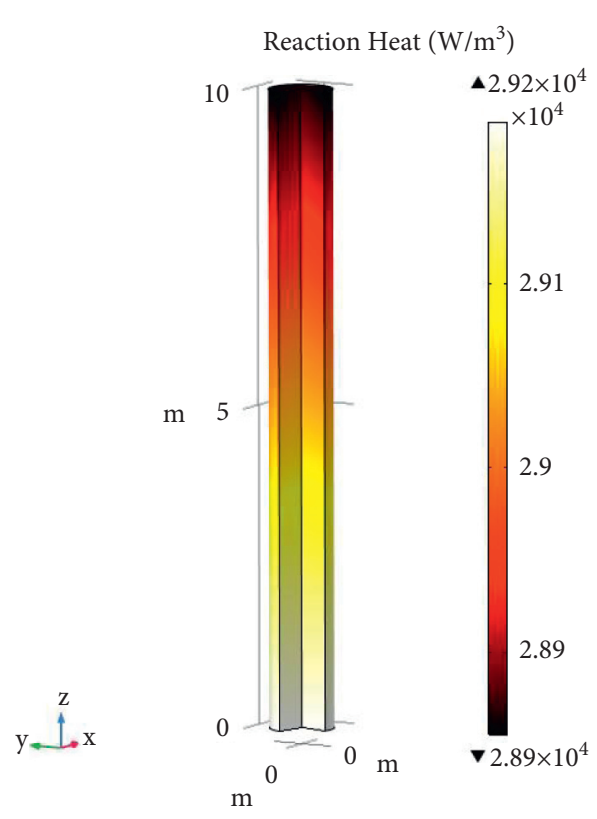

Figure 3: Heat of the reaction.

The increasing pellet diameter results in decreasing product purity. The phenomenon occurs because the catalyst mass decreases with increasing catalyst size. This reduces the active sites of the catalyst so that the conversion decreases. More reactants presented in the reacting mixture decrease the product purity. However, within the low range of the pellet diameter, the increase in the pellet diameter provides better mixing in the bed interstice. The reactant molecules in the liquid phase reach the external surface of the catalyst particles more easily as the bed porosity increases. The highest product purity was achieved with the catalyst pellet diameter at $1 \mathrm{~mm}$.

Based on simulations with various inlet gas velocities, the highest product purity was achieved at $0.5 \mathrm{~cm} / \mathrm{s}$. When the velocity increases, the residence time reduces, which results 


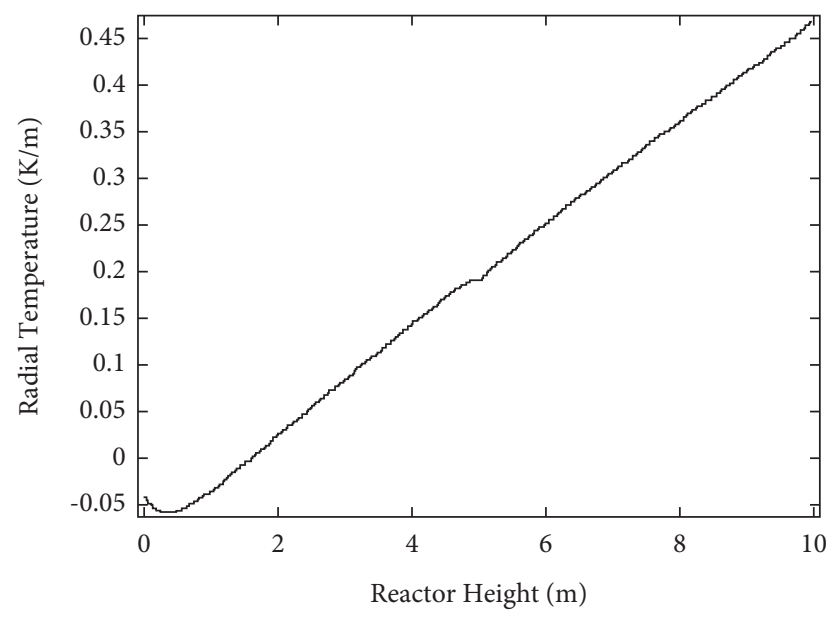

FIgURE 4: Radial temperature gradient along the reactor.

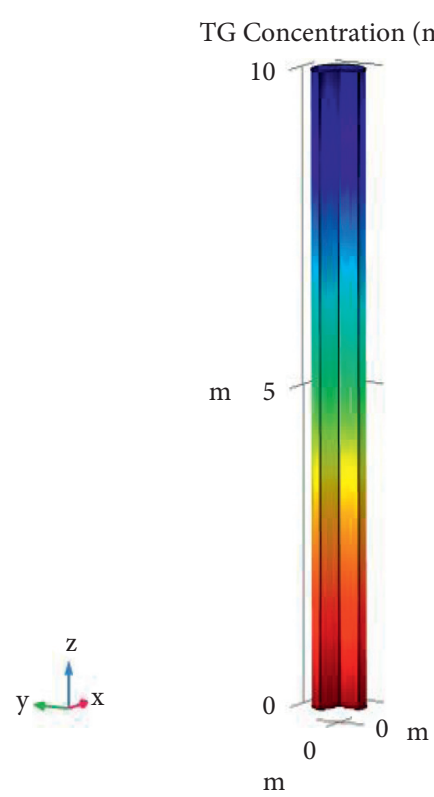

(a)

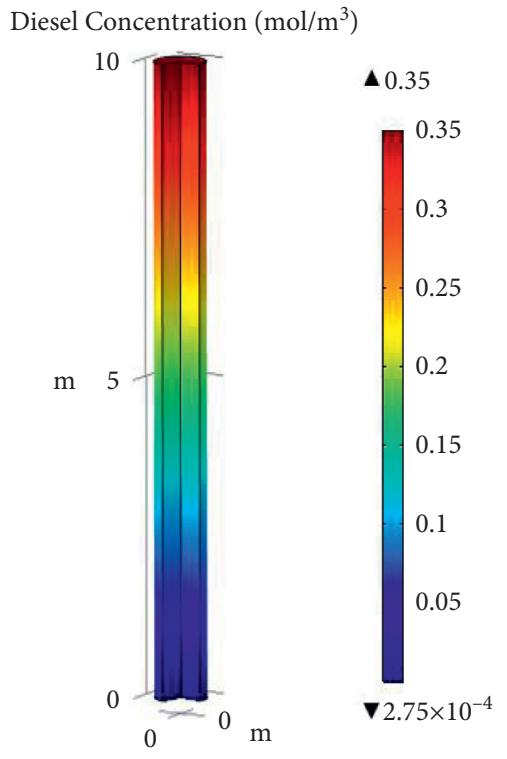

m

(b)

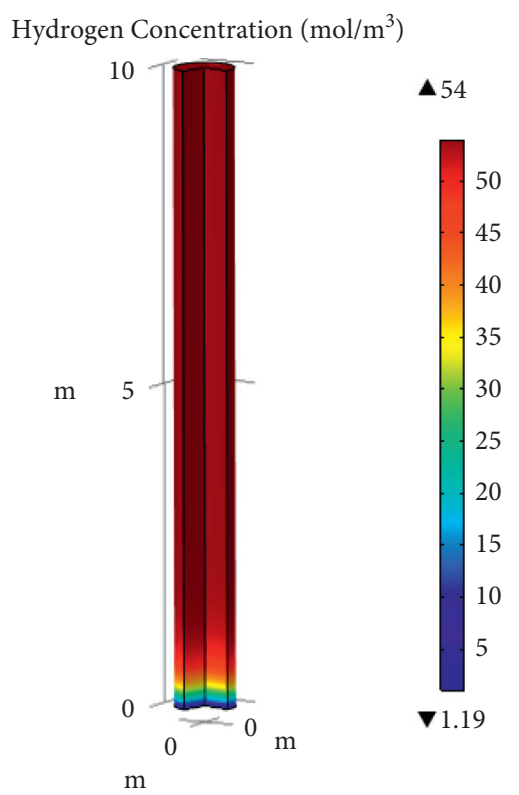

(c)

Figure 5: Concentration profiles of (a) triglyceride, (b) diesel, and (c) hydrogen. 


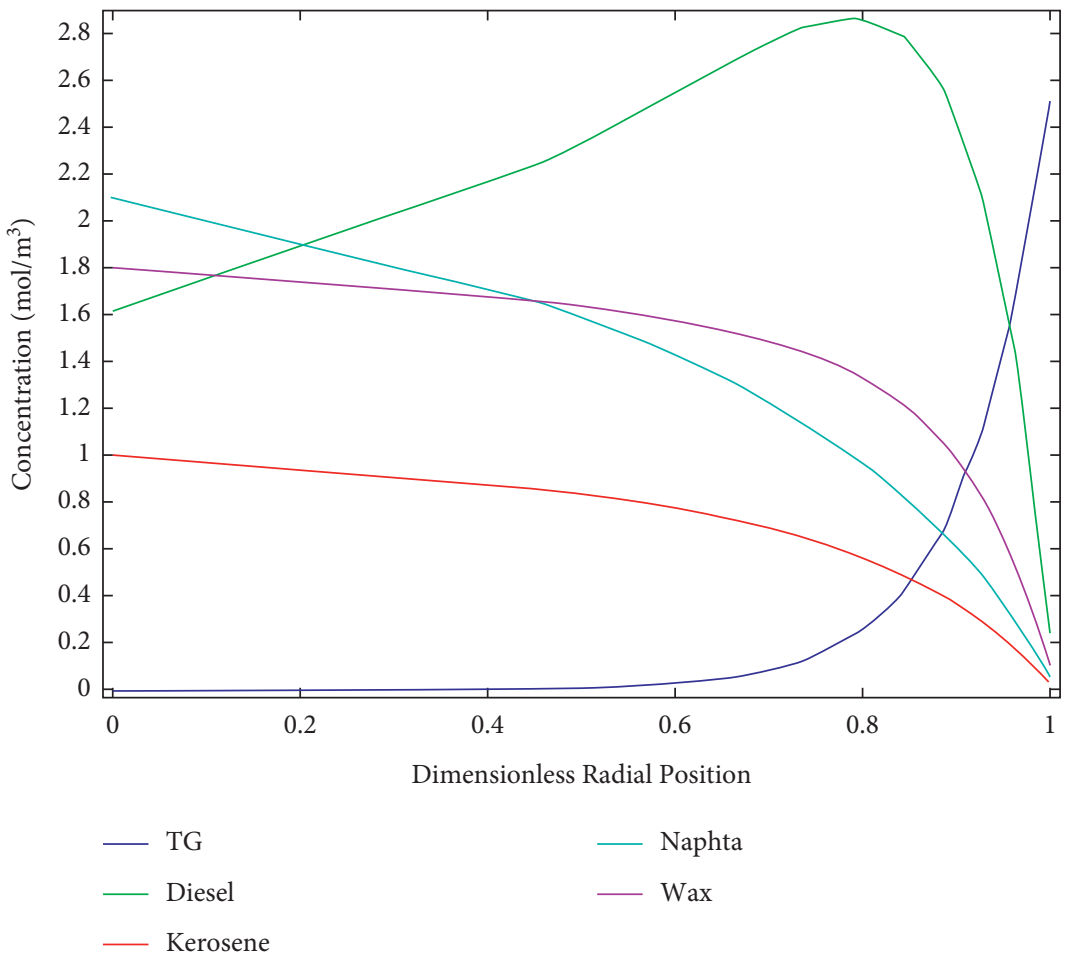

FIGURE 6: Concentration profiles in the catalyst pellet.

Table 5: Process parameter ranges.

\begin{tabular}{lc}
\hline Parameter & Range \\
\hline Particle diameter $(\mathrm{mm})$ & $0.5-10$ \\
Inlet gas velocity $(\mathrm{cm} / \mathrm{s})$ & $0.5-9$ \\
Feed molar ratio & $21.1-105.5$ \\
Inlet temperature $\left({ }^{\circ} \mathrm{C}\right)$ & $340-500$ \\
\hline
\end{tabular}

in reduced conversion. Thus, the product purity also decreases. Although the highest purity was obtained at the lowest velocity in the simulation range, the production rate was low at a low velocity. Therefore, the best velocity is not at $0.5 \mathrm{~cm} / \mathrm{s}$ but at $1 \mathrm{~cm} / \mathrm{s}$. At this velocity, the product purity remains high, and the production rate is not too low.

Changing the feed molar ratio changes the feed triglyceride concentration since the simulation was conducted with excess hydrogen. Therefore, a higher feed molar ratio means less triglyceride in the feed and more hydrogen in the feed. Since triglyceride is the limiting reactant, the increase in the hydrogen concentration induces an increase in the converted triglyceride; thus, the product purity increases. The best feed molar ratio is 105.5, which is the highest ratio in the simulation range.

Since the reaction kinetics are affected by the temperature, the reaction temperature has an interesting effect on the product purity. Generally, an increase in the reaction temperature causes an increase in product purity. After $420^{\circ} \mathrm{C}$, however, the purity of diesel decreases, while the purities of kerosene and naphtha increase exponentially. This is because diesel is cracked to become kerosene and naphtha at higher reaction rates above $420^{\circ} \mathrm{C}$. The diesel purity reached $43.26 \%$ at $420^{\circ} \mathrm{C}$, with the green fuel purity being $51 \%$. The green fuel purity increases up to $69 \%$ at $500^{\circ} \mathrm{C}$. The purities of individual diesel, kerosene, and naphtha at $500^{\circ} \mathrm{C}$ are shown in Table 6 . The hydrocracking reaction leads to hydrocarbon products with shorter chains at higher temperatures.

The optimisation results are resumed in Table 7. The table shows that if diesel purity is emphasised, then the inlet gas velocity must be $1 \mathrm{~cm} / \mathrm{s}$, the catalyst diameter must be $1 \mathrm{~mm}$, the feed molar ratio must be 105.5, and the inlet temperature must be $420^{\circ} \mathrm{C}$. This gives the $43.26 \%$ diesel purity and $44.57 \%$ yield. This table also provides information on when the purities of kerosene or naphtha are emphasised.

These optimisation results were then compared to the optimisation results of the triglyceride hydrotreating process to produce green diesel in a TBR through simulations conducted by Muharam et al. [16]. These results were also compared to the experimental results obtained by Anand et al. [14] for the kinetics study, whose models were used in this study. The comparative information on the three studies is shown in Table 8 .

The table shows that the conversion and yield of the hydrocracking process are higher than those of the hydrotreating process. This is because the hydrocracking process occurs at a higher temperature. In addition, the energy supplied to the reacting mixture breaks down more chemical bonds of reactants in hydrocracking than in hydrotreating. In the hydrotreating process, many bond breakings occur in double bonds or other weak bonds.

The product purity in this study cannot be compared to that in the hydrotreating process because of differences in purity calculations. In the hydrotreating process, the purity was calculated by involving a solvent, which was assumed to have the same properties as diesel products. 

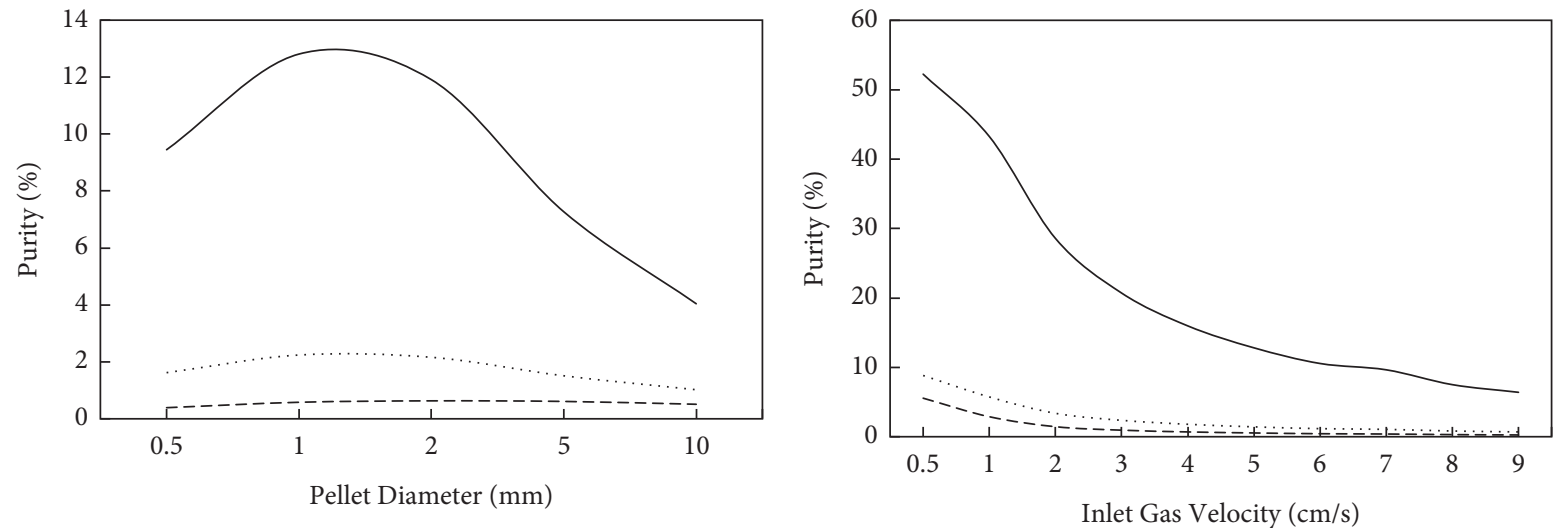

$$
\begin{aligned}
& \text { — Diesel Purity } \\
& \text {--- Kerosene Purity } \\
& \text { … Naphta Purity }
\end{aligned}
$$

$$
\begin{aligned}
& \text { — Diesel Purity } \\
& \text {--- Kerosene Purity } \\
& \ldots . . \text { Naphta Purity }
\end{aligned}
$$

(a)
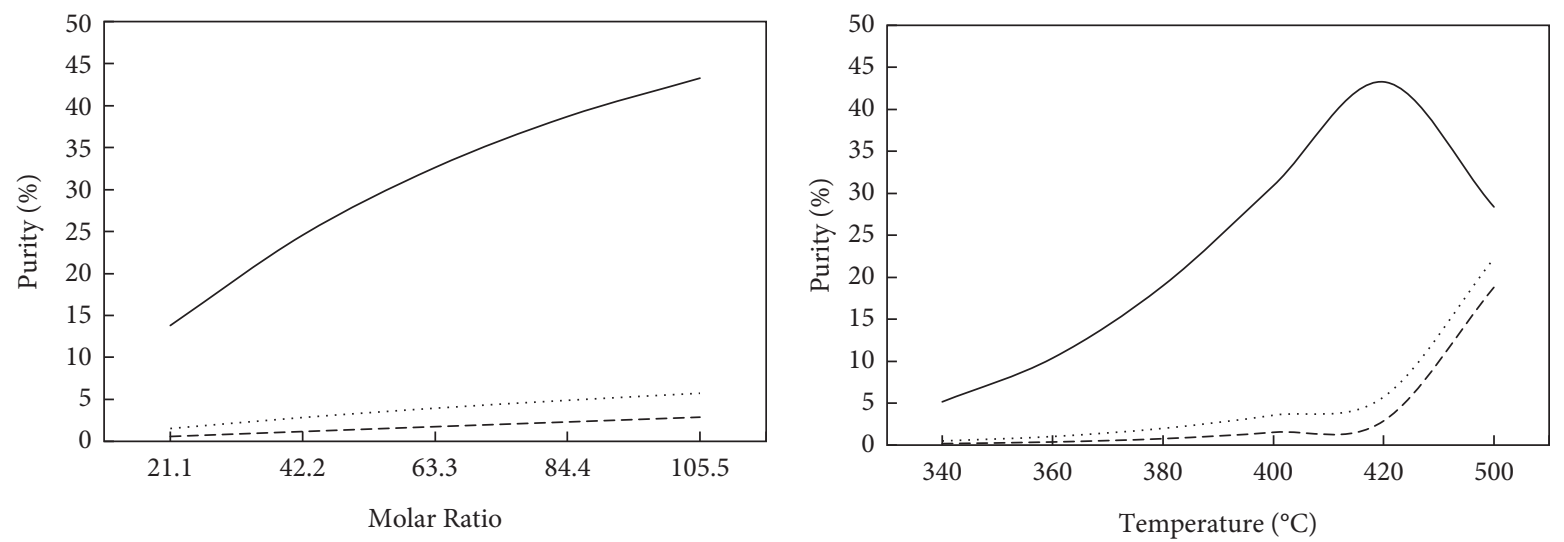

$$
\begin{aligned}
& \text { — Diesel Purity } \\
& \text {--- Kerosene Purity } \\
& \text { … Naphta Purity }
\end{aligned}
$$

— Diesel Purity

--- Kerosene Purity Naphta Purity

(d)

FIgURe 7: Process parameters of optimisation results: (a) particle diameter, (b) gas inlet velocity, (c) feed molar ratio, and (d) inlet temperature.

Table 6: Purity of products at $500^{\circ} \mathrm{C}$.

\begin{tabular}{lr}
\hline Product & Purity (\%) \\
\hline Diesel & 28.37 \\
Kerosene & 18.8 \\
Naphtha & 22.24 \\
Total & 69.41 \\
\hline
\end{tabular}

TABLE 7: Optimisation results based on the purity of each product.

\begin{tabular}{lccc}
\hline Parameter & Diesel product & Kerosene product & Naphtha product \\
\hline Catalyst diameter $(\mathrm{mm})$ & 1 & 1 & 1 \\
Inlet gas velocity $(\mathrm{cm} / \mathrm{s})$ & 1 & 1 & 1 \\
Feed molar ratio & 105.5 & 105.5 & 105.6 \\
Inlet temperature $\left({ }^{\circ} \mathrm{C}\right)$ & 420 & 500 & 500 \\
Yield $(\%)$ & 44.57 & 21.52 & 25.46 \\
Purity $(\%)$ & 43.26 & 18.80 & 22.24 \\
\hline
\end{tabular}


TABLE 8: Comparison of this study's results with Muharam et al. [11] and Anand et al. [14].

\begin{tabular}{lccc}
\hline Parameter & This research & Muharam et al. [11] & Anand et al. [14] \\
\hline Process & Hydrocracking & Hydrotreating & Hydrocracking \\
Reactor diameter $(\mathrm{cm})$ & 10 & 150 & 0.65 \\
Reactor height $(\mathrm{cm})$ & 1000 & 800 & 3.5 \\
Catalyst diameter $(\mathrm{mm})$ & 1 & 1.6 & 1.5 \\
Inlet gas velocity $(\mathrm{cm} / \mathrm{s})$ & 1 & 188 & $8.00 E-04$ \\
Feed molar ratio & 105.5 & $5 \% w / w$ & 63.3 \\
TG fraction in feed & $5 \% w / w$ & 375 & $5 \%$ \\
Inlet temperature $\left({ }^{\circ} \mathrm{C}\right)$ & 500 & 34.5 & 420 \\
Pressure (bar) & 80 & 88.3 & 80 \\
Triglyceride conversion $(\%)$ & 99.97 & 58.5 & 100 \\
Yield (\%) & 79.46 & $97.8 *$ & 87.43 \\
Purity $(\%)$ & 69.4 & & N/A \\
\hline
\end{tabular}

${ }^{*}$ Calculation involved solvent.

When compared to the experimental results in the small reactor with a powder catalyst performed by Anand et al. [14], the yield calculated in this study is lower, with a difference of $5.54 \%$. In fact, the purity in this study is the highest purity, which is the result of optimisation. The higher energy and mass transfer resistances in the larger reactor are believed to be responsible for this difference. Advantageously, both the model and the experimental study achieved a high triglyceride conversion, $99.97 \%$ and $100 \%$, respectively.

\section{Conclusion}

The hydrocracking model of jatropha oil in a TBR was successfully developed in this study. Good heat transfer in the reactor was achieved with an aspect ratio of 100 . The diesel purity reaches $44.22 \%$ at $420^{\circ} \mathrm{C}$, kerosene purity reaches $21.39 \%$ at $500^{\circ} \mathrm{C}$, and naphtha purity reaches $25.30 \%$ at $500^{\circ} \mathrm{C}$. With varying the inlet temperature, feed molar ratio, gas inlet velocity, and catalyst diameter, it is observed that the green fuel purity increases with the increase in inlet temperature and feed molar ratio, while the green fuel purity decreases with the increase in catalyst diameter and gas inlet velocity. The optimum condition is achieved at the catalyst diameter of $1 \mathrm{~mm}$, the inlet gas velocity of $1 \mathrm{~cm} / \mathrm{s}$, the feed molar ratio of 105.5 , and the inlet temperature of $500^{\circ} \mathrm{C}$. The green fuel purity achieved at this optimum condition is $69.4 \%$ with a triglyceride conversion of $99.97 \%$.

\section{Data Availability}

The data used to support the findings of this study are included within the article.

\section{Conflicts of Interest}

The authors declare that they have no conflicts of interest.

\section{Acknowledgments}

The authors thank the University of Indonesia, which funded this research through the scheme of Publikasi Terindeks Internasional (PUTI) Q2 no. NKB-1709/UN2.RST/ HKP.05.00/2020.

\section{References}

[1] E. Furimsky, "Hydroprocessing challenges in biofuels production," Catalysis Today, vol. 217, pp. 13-56, 2013.

[2] R. S. Boyas, F. T. Zarraga, and F. D. Loyo, Hydroconversion of Triglycerides into Green Liquid Fuels, I. Karame and Hydrogenation, Eds., InTech Corporation, Mexico City, Mexico, 2012.

[3] E. Hambali, "Prospek pengembangan tanaman jarak pagar untuk biodiesel dan produk turunan lainnya," Bogor: Pusat Penelitian Surfaktan Dan Energi, LPPM-IPB, Dramaga, Indonesia, 2006.

[4] G. Biardi and G. Baldi, "Three-phase catalytic reactors," Catalysis Today, vol. 52, no. 2-3, pp. 223-234, 1999.

[5] I. Iliuta and F. Larachi, "Hydrocarbon hydrodesulfurization in vertical, inclined and oscillating trickle beds-hydrodynamics $\&$ reactor performance for offshore petroleum marine applications," Fuel, vol. 186, no. 57, pp. 35-49, 2016.

[6] A. Gianetto and V. Specchia, "Trickle-bed reactors: state of art and perspectives," Chemical Engineering Science, vol. 47, no. 13-14, pp. 3197-3213, 1992.

[7] A. K. Giri and S. K. Majumder, "Pressure drop and its reduction of gas-non-newtonian liquid flow in downflow trickle bed reactor (DTBR)," Chemical Engineering Research and Design, vol. 92, no. 1, pp. 34-42, 2014.

[8] S. A. Al-Naimi, F. T. J. Al-Sudani, and E. K. Halabia, "Hydrodynamics and flow regime transition study of trickle bed reactor at elevated temperature and pressure," Chemical Engineering Research and Design, vol. 89, no. 7, pp. 930-939, 2011.

[9] V. Russo, T. Kilpiö, M. Di Serio et al., "Dynamic non-isothermal trickle bed reactor with both internal diffusion and heat conduction: sugar hydrogenation as a case study," Chemical Engineering Research and Design, vol. 102, pp. 171-185, 2015.

[10] Y. Muharam, T. Aufa, and T. B. Santoso, "Modeling of partial hydrogenation of polyunsaturated fatty acid methyl esters in a trickle bed reactor," Engineering Journal, vol. 24, no. 4, pp. 195-204, 2020.

[11] A. Milbrandt, C. Kinchin, and R. McCormick, The Feasibility of Producing and Using Biomass-Based Diesel and Jet Fuel in the United States. Colorado, National Renewable Energy Laboratory, Denver, CO, USA, 2013.

[12] L. Attanatho, Performance and Kinetic Studies of Hydrotreating of Bio-Oils in Microreactor, Oregon State University, Corvallis, Oregon, 2012.

[13] Y. Muharam and J. A. Soedarsono, "Hydrodeoxygenation of vegetable oil in a trickle bed reactor for renewable diesel 
production," International Journal of Technology, vol. 11, no. 7, pp. 1292-1299, 2020.

[14] M. Anand, S. A. Farooqui, R. Kumar et al., "Kinetics, thermodynamics and mechanisms for hydroprocessing of renewable oils," Applied Catalysis A: General, vol. 516, pp. 144-152, 2016.

[15] Y. Muharam and O. A. Nugraha, "Prediction of the effects of the inlet velocity and the reactor length on the performance of a trickle-bed reactor for renewable diesel production," $A d$ vanced Science Letters, vol. 23, no. 6, pp. 5609-5614, 2017.

[16] Y. Muharam, O. A. Nugraha and D. Leonardi, "Modelling of a hydrotreating reactor to produce renewable diesel from nonedible vegetable oils," Chemical Engineering Transactions, vol. 56, pp. 1561-1566, 2017.

[17] A. E. Sáez and R. G. Carbonell, "Hydrodynamic parameters for gas-liquid cocurrent flow in packed beds," AIChE Journal, vol. 31, no. 1, pp. 52-62, 1985.

[18] R. M. Propp, P. Colella, W. Y. Crutchfield, and M. S. Day, "A numerical model for trickle bed reactors," Journal of Computational Physics, vol. 165, no. 2, pp. 311-333, 2000.

[19] M. C. Leverett, "Capillary behavior in porous solids," Transactions of the AIME, vol. 142, no. 01, pp. 152-169, 1941.

[20] K. Grosser, R. G. Carbonell, and S. Sundaresan, "Onset of pulsing in two-phase cocurrent downflow through a packed bed," AIChE Journal, vol. 34, no. 11, pp. 1850-1860, 1988.

[21] S. Goto and J. M. Smith, "Trickle-bed reactor performance: part I. holdup and mass transfer effects," AIChE Journal, vol. 21, no. 4, pp. 706-713, 1975.

[22] D. W. van Krevelen and J. T. C. Krekels, "Rate of dissolution of solid substances part I: rate of mass transfer in granular beds (physical dissolution)," Recueil des Travaux Chimiques des Pays-Bas, vol. 67, no. 7, pp. 512-520, 1948.

[23] C. R. Wilke and P. Chang, "Correlation of diffusion coefficients in dilute solutions," AIChE Journal, vol. 1, no. 2, pp. 264-270, 1955.

[24] C. R. Wilke and C. Y. Lee, "Estimation of diffusion coefficients for gases and vapors," Industrial and Engineering Chemistry, vol. 47, no. 6, pp. 1253-1257, 1955.

[25] M. A. Matthews, J. B. Rodden, and A. Akgerman, "Hightemperature diffusion of hydrogen, carbon monoxide, and carbon dioxide in liquid n-heptane, n-dodecane, and n-hexadecane," Journal of Chemical \& Engineering Data, vol. 32, no. 3, pp. 319-322, 1987.

[26] M. J. Taulamet, N. J. Mariani, G. F. Barreto, and O. M. Martínez, "Estimation of overall heat transfer coefficients in packed beds with cocurrent downflow of gas and liquid," Fuel, vol. 138, pp. 176-182, 2014.

[27] J. M. Hochman and E. Effron, "Two-phase cocurrent downflow in packed beds," Industrial \& Engineering Chemistry Fundamentals, vol. 8, no. 1, pp. 63-71, 1969.

[28] Aspen, Aspen HYSYS, Aspen Technology, Inc, Bedford, MA, USA, 2017. 